\title{
Description and Characterization of a ECR Plasma Device Developed for Thin Film Deposition
}

\author{
J.A.S. da Matta ${ }^{\dagger}$, R.M.O. Galvão, L. Ruchko, \\ M.C.A. Fantini, and P.K. Kiyohara \\ $\dagger$ †epartamento de Física, Centro Universitário da FEI \\ UNIFEI, 09850-901, São Bernardo, SP, Brazil \\ Instituto de Física, Universidade de São Paulo, \\ C.P. 66318, 05315-970, São Paulo, SP, Brazil
}

Received on 23 May, 2002

\begin{abstract}
The design, construction, and characterization of an electron-cyclotron-resonance (ECR) plasma device and its utilization for growing AIN polycrystals are described in detail. The plasma density and electron temperature were measured by two types of Langmuir probes under different conditions of magnetic configuration and $\mathrm{RF}$ substrate polarization. For the investigated nitrogen plasmas, the electron temperature increases towards substrate holder and decreases with pressure. The magnetic configuration and plasma parameters required for successful growth of polycrystal aluminum nitride have been determined.
\end{abstract}

\section{Introduction}

Microwave plasma sources are being used increasingly for thin film deposition and etching [1]. In particular, plasmas produced by resonant absorption at the electron cyclotron frequency, in the so-called ECR (electron-cyclotron resonance) devices offer a number of desirable characteristics, including high plasma density, low pressure operation, efficient gas utilization, and uniform plasma with a high degree of ionization and decomposition of working gases [2]. Due to the electrodless nature of ECR plasma production, it is possible to obtain independent control of ion energy and flux by application of a low-frequency RF bias to the substrate [3]. Although ECR devices are now commercially available, they are usually designed for special applications and the user has little access for controlling and characterizing the plasma conditions. In this work we describe an ECR device designed and build at the Plasma Laboratory of the Institute of Physics of University of São Paulo. The basic configuration is standard, with the magnetic field produced by a two coil system. The main characteristics of the plasma, in both mirror and divergent magnetic field configurations have been determined, as well as the necessary plasma conditions for optimized growth of aluminum nitride crystals.

\section{Description of the ECR Device}

The diagram of the ECR device and a photo of the plasma in the reaction chamber are shown in Figs. 1 and 2, respectively. The microwave power ( $2.45 \mathrm{Ghz}, 1.3 \mathrm{~kW}$ maximum) is provided by a home-made source using a conventional magnetron from commercial microwave ovens. The microwave is guided to the vacuum chamber through a rectangular waveguide, in the $T E_{01}$ mode, with a three-port circulator and a stub-turner section. Vacuum seal is provided by a fused quartz window between the straight and bent waveguide sections. The bent section is used to avoid that the plasma streaming out along the magnetic field lines strikes directly the quartz window. However, we have found that, in long operations, some plasma may still strike the window causing localized heating and material damage. This will be corrected in the future by placing the vacuum window further away from the plasma. Right before the vacuum chamber, a mode conversion section is used to convert the $T E_{01}$ mode to a $T E_{11}$ circularly polarized one. The microwave incident power $P_{i}$ and reflected power $P_{r}$, are monitored by a microwave powermeter.

The magnetic field is generated by two coils, made of copper conductors of $1 \mathrm{~cm}^{2}$ cross-section with a central hole of diameter $5 \mathrm{~mm}$, for water-cooling. The coils are assembled from two-layer modules, each one having inner diameter $51 \mathrm{~cm}$, with 10 turns in each layer. The cooling system is connected in parallel between the different modules and coils are electrically connected in series. They are fed by a three-phase conventional rectifier, which can deliver a maximum current of $250 \mathrm{~A}$, at $20 \mathrm{~V}$.

The magnetic field configuration can be changed by varying the distance between the coils. In Fig. 3 we show the axial profile of the magnetic field as a function of the coil separation, normalized to the value of the magnetic field at the electron-cyclotron resonance, $B=875 G$. For each configuration, the position of the resonant layer can be ad- 
justed to some extent by varying the currents in the coils. For a coil separation $d=15 \mathrm{~cm}$, for instance, the resonant field requires a coil current $I_{B}=180 \mathrm{~A}$. The transition between divergent and mirror field profiles is obtained for a coil separation $d=10 \mathrm{~cm}$, approximately.

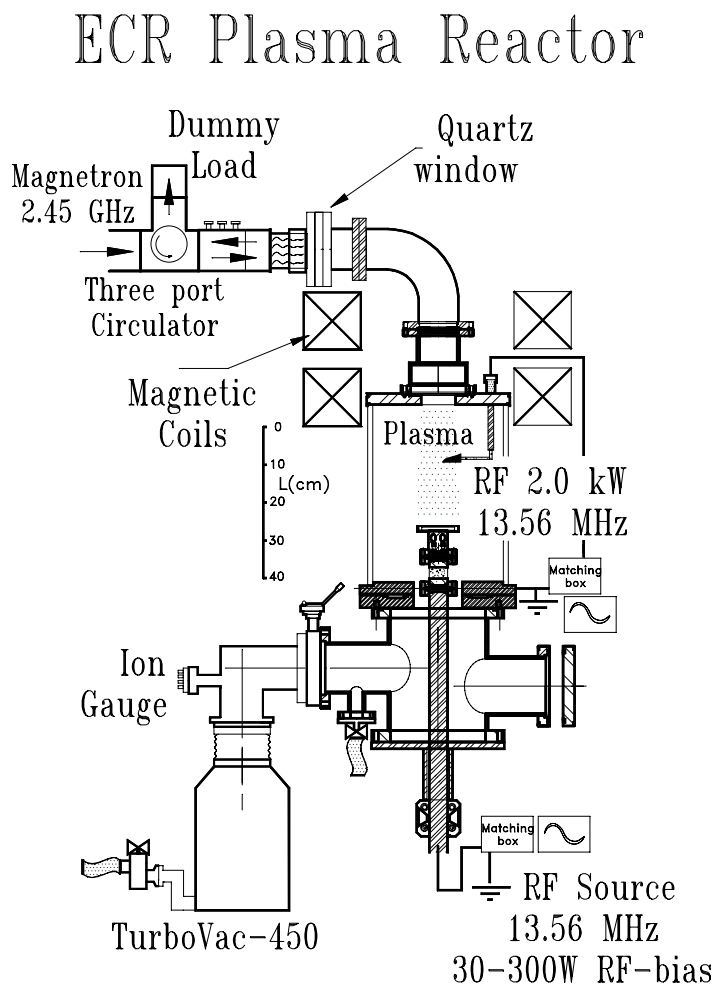

Figure 1. Schematic diagram of the ECR device.

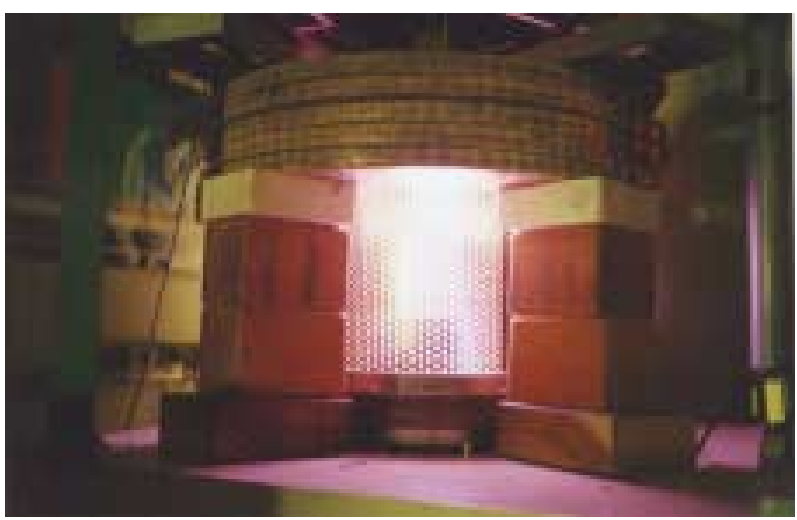

Figure 2. Photo of the plasma inside the pyrex vacuum chamber.

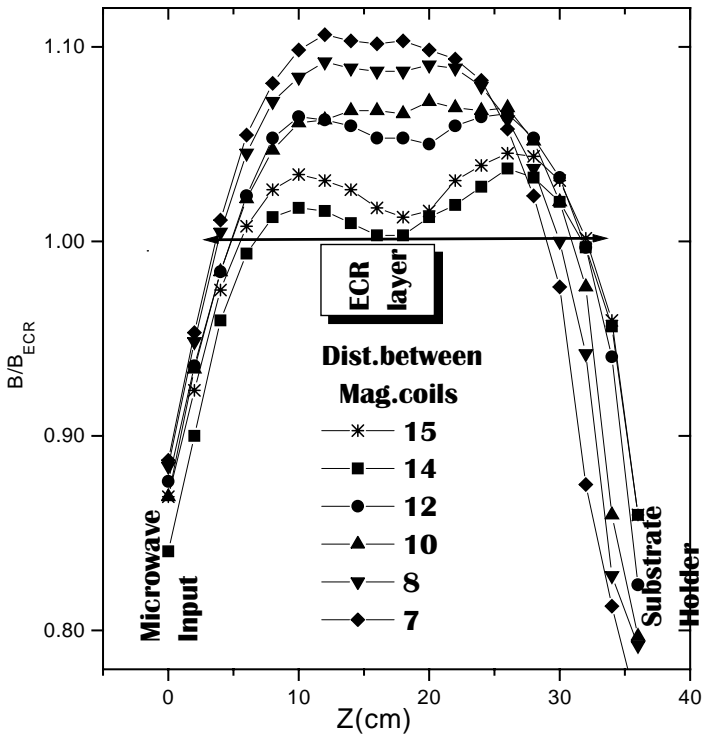

Figure 3. Axial profiles of the normalized magnetic field for different coil separations $(7-15 \mathrm{~cm})$.

The basic vacuum conditions are produced by a $450 \mathrm{l} / \mathrm{s}$ turbo-molecular pump. The base pressure is $10^{-6} \mathrm{mbar}$ and the operating pressure is varied within the range $10^{-4}-$ $10^{-5}$ mbar for nitrogen and argon plasmas. The substrate holder has a tantalum plate of $\phi 7.6 \mathrm{~cm}$ diameter, which, in the presence of the plasma, can be dynamically polarized to approximately $-120 \mathrm{~V}$, by an independent $300 \mathrm{~W}$ RF power supply. The maximum RF power density delivered to the substrate holder is $6.6 \mathrm{~W} / \mathrm{cm}^{2}$. Another more robust RF power supply is available for sputtering source samples, if desired. It can deliver up to $2.0 \mathrm{~kW}$ of RF power and provide a self-polarization of metal samples up to $-400 \mathrm{~V}$, in normal plasma operating conditions. The sample holder is connected to an isolated metal rod, inserted into the vacuum chamber through a dynamic vacuum feedthrough, located at the upper flange of the vacuum chamber, so that the sample can be moved in and out of the plasma by axial rotation.

\section{II.1 Plasma Parameters}

A movable single electrostatic probe is used to measure the electron temperature, plasma density, and plasma potential. The probe is assembled inside a stainless steel tube, which is inserted into the vacuum chamber through the same dynamic feedthrough that is employed for the holder of the source sample. Therefore, the measurements cannot be made simultaneously with RF sputtering. The probe bias is usually sweeped from $-100 \mathrm{~V}$ to $+100 \mathrm{~V}$. An especially designed RF-shielded probe, which will be discussed in the sequel, is used for operation with RF polarization of the substrate. 


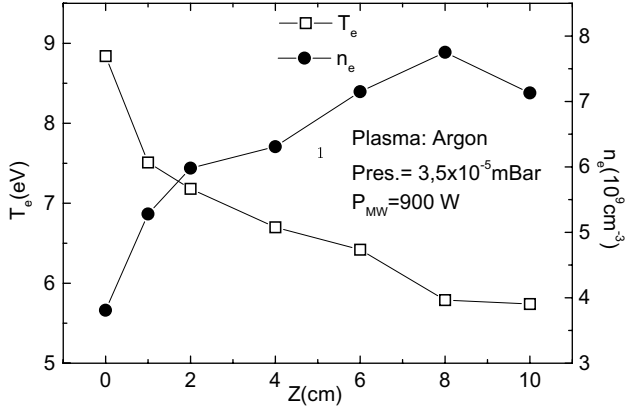

(a)

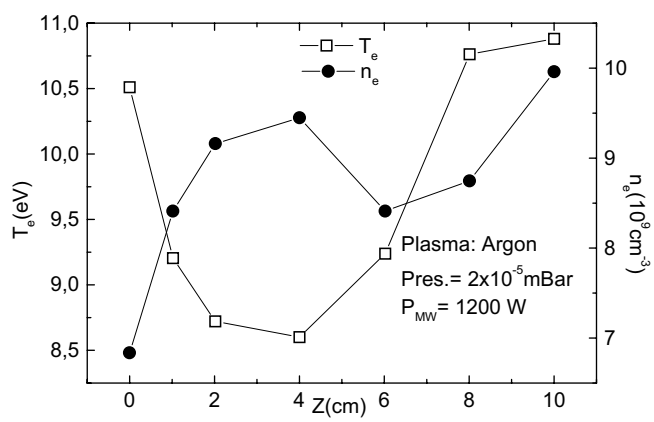

Figure 4. ECR plasma profiles. Mirror (a) and divergent (b) magnetic configurations.

The axial profiles of the electron temperature and plasma density are show in Fig. 4 for an argon plasma and for mirror (a) and divergent (b) magnetic field configurations, without RF polarization. The distances are measured from the surface of the substrate. For the mirror field configuration, the lowest resonant layer is located at $z=15 \mathrm{~cm}$ and for divergent field at $z=25 \mathrm{~cm}$. It has been found that the optimization of the parameters (temperature, density, and quiescence) depends on the neutral gas pressure and on the magnetic field configuration. Actually, the neutral pressure dependence has been already discussed in other works [4]. However, the dependence on the magnetic field configuration has not yet been investigated in detail. Accordingly, the neutral gas pressure is $3.5 \times 10^{-5}$ mbar for the mirror configuration, Fig.4a, and $2.0 \times 10^{-5}$ mbar for the divergent-field configuration, Fig.4b.

These pressures are much below the range in which the ECR devices are usually operated, approximately from $10^{-4}$ to $10^{-2}$ mbar [4]. Therefore, the results presented in this paper provide an extension of the plasma parameters of ECR devices towards the collisionless regime.

To avoid self polarization of the electrostatic probe, when operating in the presence of RF fields, a compensated probe has to be used. In Fig.5 we show the scheme of a probe constructed for this purpose according to the general concepts discussed by Castro et al. [5]. The miniaturized discrete components of the filter circuit are assembled inside a curved pyrex tube that connects the probe tip to the holder. The resonant circuit, made by the inductor $L_{1}+L_{2}$ in parallel with the capacitor $C_{1}$, is tuned to the frequency 13.56 $\mathrm{MHz}$. The capacitor $C_{0}$ provides a RF ground to the compensating electrode around the probe tip.

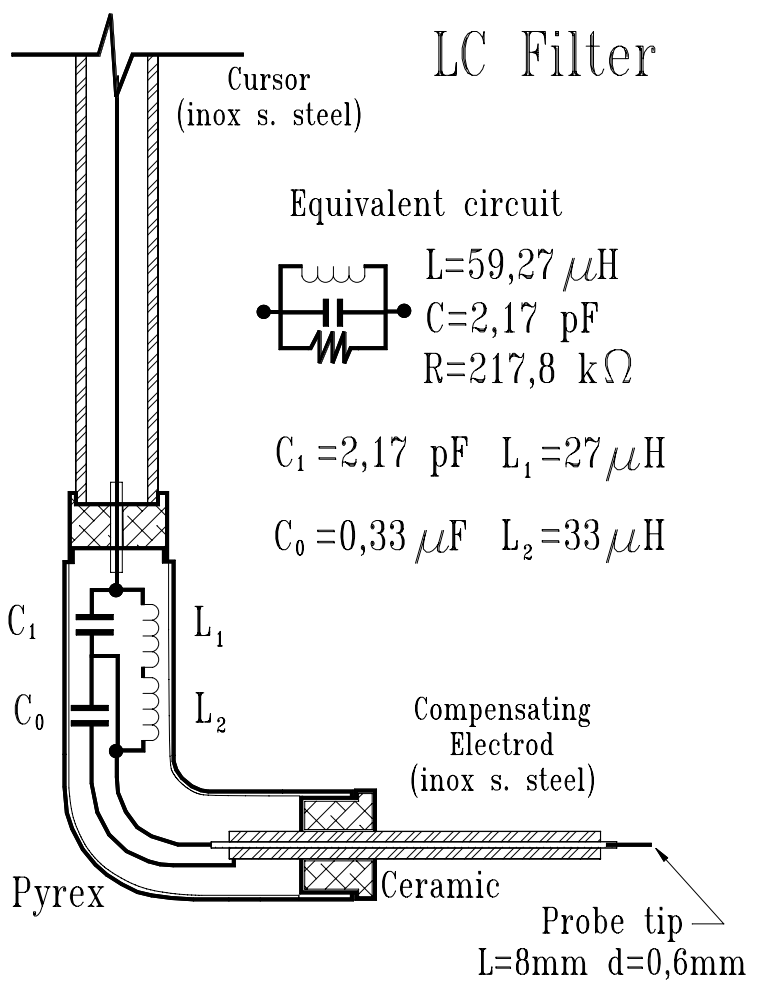

Figure 5. Compensating electrostatic probe.

The operation with RF polarization of the substrate was carried out in a $N_{2} / A r(80 \% / 20 \%)$ plasma, at a somewhat higher neutral pressure, $p=5.0 \times 10^{-5}$ mbar. Only the mirror configuration was used and the microwave power was increased to $1.1 \mathrm{~kW}$. In Fig.6 we show the axial profiles of the electron temperature and plasma density for two levels of RF power, namely $30 W$ (a) and $60 W$ (b). Although the profiles are similar to the ones for the mirror configuration without RF (Fig.4a), the temperature raise towards the substrate is much steeper and the temperature close to the surface of the substrate increases with the RF power. For $P_{R F}=60 \mathrm{~W}$, the self-polarization of the substrate reaches $\left\langle V_{R F}\right\rangle \sim-100 \mathrm{~V}$ increasing substantially the ion acceleration towards it.

A summary of the plasma conditions explored in this device, for filling neutral densities below $4.0 \times 10^{-5}$ mbar is given in Table 1.

\begin{tabular}{lrr}
\hline Magnetic Config. & $T_{e}(\mathrm{eV})$ & $n_{e}\left(10^{9} \mathrm{~cm}^{-3}\right)$ \\
\hline Mirror & $7-15$ & $5-20$ \\
Divergent & $8-11$ & $6-12$ \\
Mirror/RF & $9-26$ & $4-20$ \\
\hline
\end{tabular}




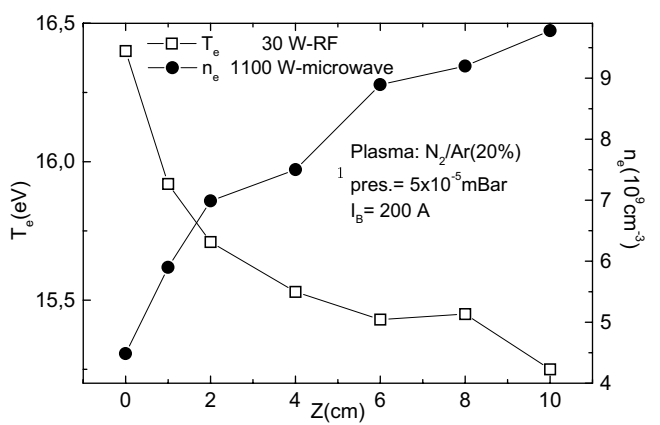

(a)

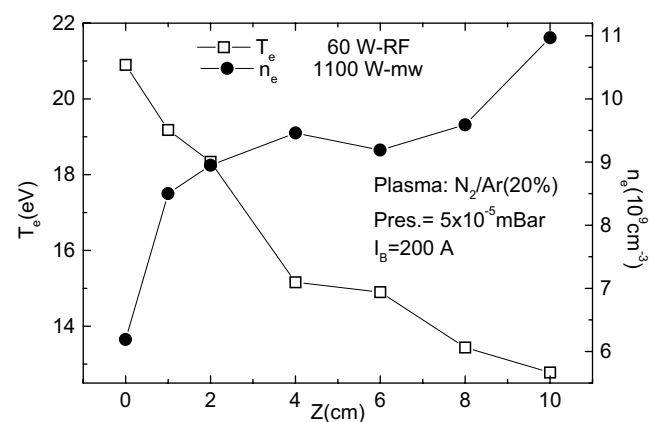

Figure 6. ECR-RF plasma profiles; N2 (Ar-20\%); (a) $30 \mathrm{~W}-\mathrm{RF}$ (b) 60W-RF.

\section{II.2 Analysis of the Results}

The results shown in Figs. 4 and 6 are rather surprising. From a simple-minded picture, we expect the electron temperature to be the highest in the resonance region where microwave power is absorbed and to decrease monotonically away from it. Since the plasma flow towards the substrate occurs along divergent field lines, simple flux conservation shows that the plasma density should decrease monotonically [6]. This is indeed observed for the mirror configuration, Figs. $4 a, 6 a$ and $6 \mathrm{~b}$, but not for the divergent field configuration, Fig.4b. Taking the maximum neutral pressure that has been used $p_{n} \sim 5.0 \times 10^{-5}$ mbar, we find that the mean-free-path for electron-neutral collisions, which is the dominant collisional mechanism for the experimental conditions, is of the order of $0.7 \mathrm{~m}$, much larger than the machine dimensions. Therefore, the electron flow is collisionless and, due to energy and magnetic moment conservation, the perpendicular energy should be converted to parallel energy as the flow proceeds towards the substrate. Actually, numerical simulations, including the ambipolar electric potential, and experimental results indicate that the electron temperature should be almost constant along the magnetic field lines [7, 8]. From these simple arguments and known results available in the literature, it follows that indeed the temperature profiles shown in Figs. 4 and 6 are rather anomalous.

In our axial measurements, along magnetic field lines under the mirror configuration with RF bias polarization, we observe a sensitive plasma potential drop as the Langmuir probe gets closer to the substrate ( $3 \mathrm{~cm}$ above), while in the mirror configuration without RF bias polarization, $V_{p}$ remains almost constant along the plasma axis. The spatial profiles of plasma potential, $V_{p}$, shown in Fig.7, indicate this behavior. This effect is in accordance with the proposed plasma heating mechanism which describe the electron motion under the $\mu \nabla B$ force by converting the parallel to perpendicular energy [10].

\section{II.3 Application}

As a simple test to characterize the performance of the ECR device for technological applications, we have tried to grow polycrystalline aluminum nitride, $A l N$, on grains of aluminum powder spread over a silicon substrate. Although $A l N$ films have already been grown in ECR devices, the techniques that have been used are somewhat different from ours; in particular employing complex gas mixtures ( $\left.\mathrm{NH}_{3} / T M A\right)[9]$.

The discharges were carried out in a $N_{2} / A r$ gas mixture; argon was introduced into the discharge mainly to help cleaning off the $\mathrm{Al}_{2} \mathrm{O}_{3}$ film on the surface of the aluminum grains. The microwave power coupled to the plasma was kept at $1.1 \mathrm{~kW}$ level. Distinct discharges conditions and magnetic field configurations were tried and the best results were obtained for a working pressure $p=5 \times 10^{-5}$ mbar, in a divergent magnetic field configuration. The substrate was positioned $25 \mathrm{~cm}$ below the resonance region.

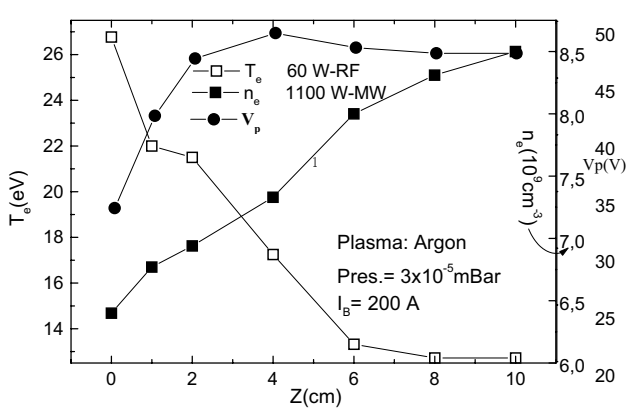

(a)

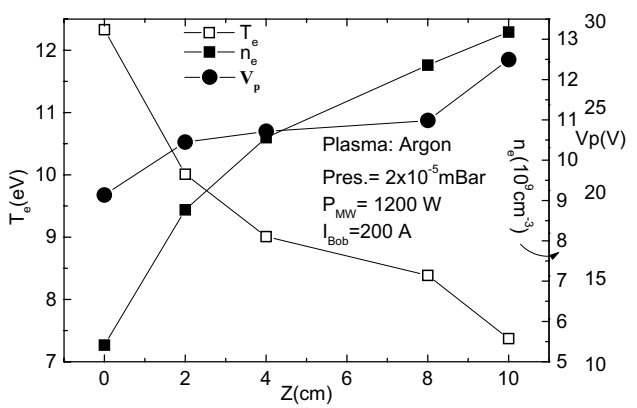

(b)

Figure 7. ECR plasma profiles; Ar; (a) $60 \mathrm{~W}-\mathrm{RF}$ (b) $0 \mathrm{~W}-\mathrm{RF}$. 
In Fig. 8 we show a scanning electron microscopy (SEM) image of the early phase of the nitride growth. The crystals are rather small and oriented in different directions. The crystals grown after $4.5 h$ exposition to the plasma are shown in Fig.9. Stalactite structures have been formed with crystal sizes of the order of $10 \mu \mathrm{m}$.

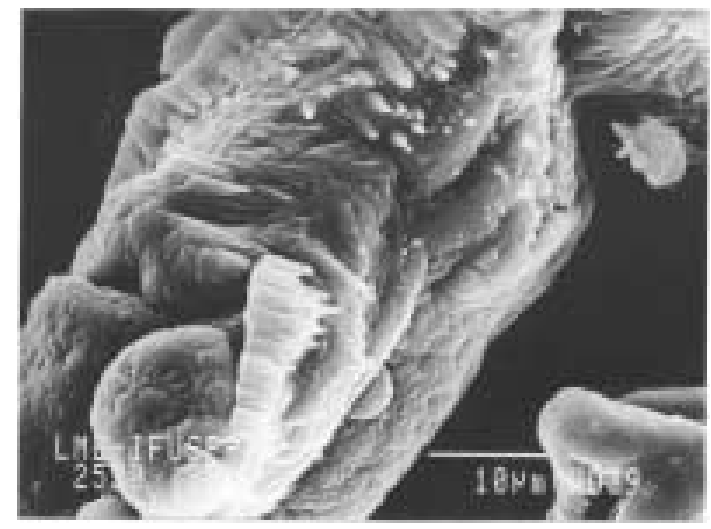

Figure 8. SEM image, grains formation.

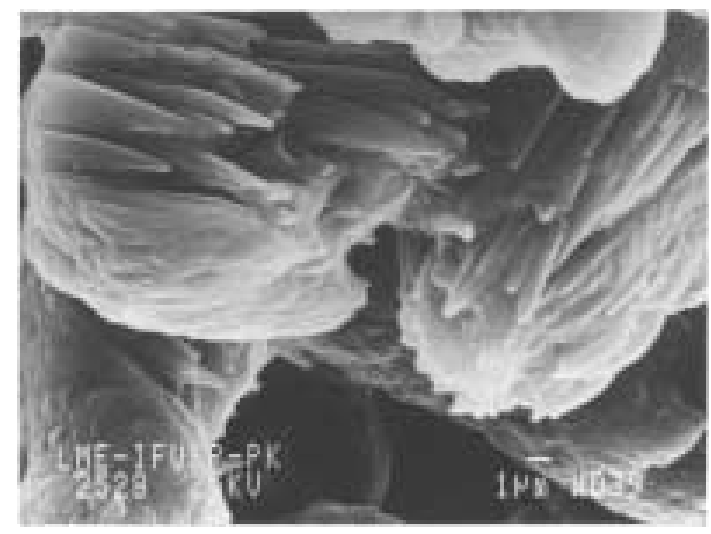

Figure 9. SEM image, stalactites formation.

The results of X-ray diffraction (XRD) analysis of the grains are shown in Fig.10. The peaks associated with $A l N$ are clearly shown, with preferential growth in the (002) orientation, as obtained by Yasui and co-workers using a different technique. More careful controlled experiments comparing $A l N$ thin film growth on different substrates is currently being carried out.

\section{Conclusion}

A description of the first ECR device entirely designed and built in Brazil has been presented. The plasma parameters obtained in the region just above the substrate are somewhat unusual in that the electron temperature increases towards

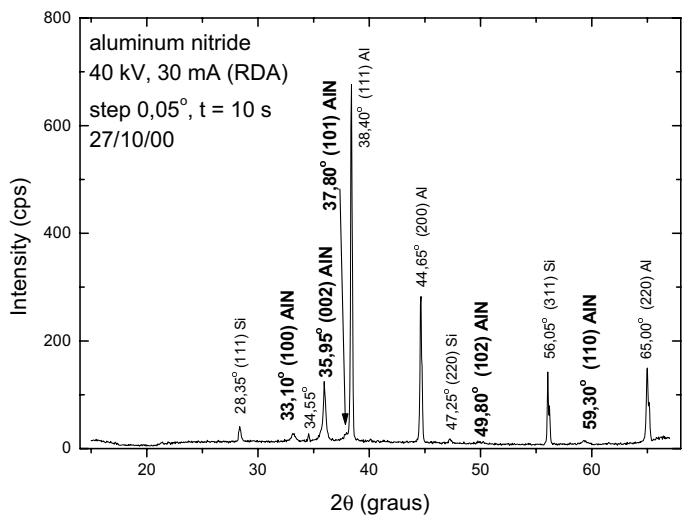

Figure 10. XRD analysis of the aluminum grains.

the substrate. A tentative explanation of these results has been discussed. The device has been successfully employed to grow $A l N$ crystals in a simple $N_{2} / A r$ plasma.

\section{Acknowledgments}

This work has been supported by " Fundação de Amparo à Pesquisa do Estado de São Paulo" and "Conselho Nacional de Pesquisas e Desenvolvimento", through the PRONEX Project. The authors are thankful to the LME, Laboratory of the Polytechnic School of University of São Paulo and to Professor M.Martins for lending some components of the microwave equipment.

\section{References}

[1] B. Chapman, Glow Discharge Processes, (1980).

[2] Y. Kawai and Y. Ueda, Surf. Coat. Technol. 12-19 (2000) 131.

[3] M.A. Lieberman and R.A. Gottscho, Design of High-Density Plasma Sources for Materials Processing, Physics of Thin Films, vol. 18, edited by M.H. Francombe e J.L. Vossen (Academic Press, 1994).

[4] J.S. Lille, P.E. McGaughnea, IEEE Transactions on Plasma Science 29, 417 (2001).

[5] R.M. Castro et al, Contrib. Plasma Phys. 39, 235 (1999).

[6] W.L. Manheimer, R.F. Fernsler, IEEE Trans. on Plasma Science 29, 75 (2001).

[7] M. Lampe, G. Joyce, W. Manheimer, S. Slinker, IEEE Transactions on Plasma Science 26, 1592 (1998).

[8] C. Eddy, D. Leonhardt, S. Douglas et. al. J. Vac. Sci. Technology A7, 883 (1989).

[9] K. Yasui, S. Hoshino and T. Akahane, Appl. Surf. Sci. 462467 (2000) 159.

[10] M. Zintl, R. McWilliams and N. Wolf, Phys. Plasmas, 2, 4432 (1995). 